\title{
Zur Bestimmung des Milchgehaltes von Milchschokoladen.
}

\author{
Vou \\ Dr. J. Groffeld in Recklinghausen. \\ (Vorläufige Mitteilung.) \\ [Eingegangen am 24. September 1922.]
}

Die Bestimmung des Milchgehaltes von Milchschololaden zergliedert sich in die Feststellung der Menge des vorhandenen Milchfettes und die Ermittelung der vorhandenen Milchtrockensubstanz.

Das Milchfett ergibt sich in einfachster Weise aus dem Gehalte der Schokolade an Gesamtfett und aus der Reichert-MeiBl'schen Zahl desselben, nach einem Verfahren, das im einzelnen von O. Laxa ${ }^{1}$ ) sowie von E. Baier und P. Neumann ${ }^{2}$ ) ausgearbeitet worden ist, worauf hier verwiesen sei. Die Bestimmung des gesamten Fettes erfolgt sehr zweckmäßig, indem man $50 \mathrm{~g}$ geraspelte Schokolade in einer großen Extraktionshülse im Soxhlet'schen Extraktionsapparate mit Äther auszieht, das ausgezogene Fett zur Wägung bringt und $5 \mathrm{~g}$ davon zur Bestimmung der Reichert-MeiBl'schen Zahl verwendet, während der entfettete Rückstand nach dem Verdunsten des Äthers für die Prüfung der Scbokolade auf weitere Bestandteile dient; besonders die polarimetrische Zuckerbestimmung kann auf einfache Weise darin vorgenommen werden, wobei die in der Schokolade selbst vorhandenen großen Fettmengen durch ihr Volumen nicht mehr stören. Die im Rückstande ermittelten Werte werden durch Multiplikation mit der Zahl (100 - Fettgehalt der Schokolade in \%) auf die ursprüngliche Schokolade umgerechnet.

Ist das Kakaofett mit einem an flüchtigen, wasserlöslichen Fettsäuren reichen Fremdfette verfälscht, so ist eine hohe Reichert-Meißl'sche Zahl an sich noch nicht beweisend für die Anwesenheit von Butterfett. - Neuerdings wird nach dem D.R.P. 357877 von den Ölwerken Germania in Emmerich sogar durch Veresterung der entsprechenden Fettsäuren in gleicher Mischung, wie sie im Butterfett enthalten sind, mit Glycerin ein künstliches Butterfett ${ }^{3}$ ) von genau gleichen Kennzahlen wie beim echten Butterfett hergestellt, zu dessen Nachweis die bisherigen analytischen Verfahren überhaupt versagen. - Andererseits ist eine zu niedrige Reichert-Meißl'sche Zahl in Milchschokoladenfett beweisend, daß eine hinreichende Menge Milchfett nicht vorhanden ist.

Die fettfreie Milchtrockensubstanz, bezw. deren Bestandteile, das Milchprotein, den Milchzucker und die Milchasche leiten die genannten Forscher aus dem Caseingehalt der Schokolade her, zu dessen Bestimmung zuerst Laxa Ammoniumoxalat verwendet, während Baier und Neumann, die das Verfahren von Laxa in mehrfacher Hinsicht verbessert und vereinfacht haben, das Casein in zweckmäßiger Weise durch Natriumoxalat ausziehen und dann durch Uranacetat in schwach essigsaurer Lösung fällen.

1) Diese Zeitschrift 1904, 7, 471.

2) Diese Zeitschrift 1909, 18, 13.

3) Dieses künstliche Butterlett wird sicher auch bei der Beurteilung von anderen milchfetthaltigen Nahrungsmitteln von Bedeutung werden und die bisher gebräuchlichen Untersuchungsverfahren in vielen Fälen wertlos machen. 
Bei früheren Untersuchungen über Milchbackwaren ${ }^{1}$ ) hatte ich darauf hingewiesen, daß der hohe Calciumoxydtgehalt der Milchasche für die Feststellung der fettfreien Milchtrockenmasse in Milchzubereitungen besonders geeignet ist. Da vor allem der Kalkgehalt der Milch, zumal in der weiter unten beschriebenen Weise, sich erheblich einfacher ermitteln läßt als der Caseingehalt, so schien es wertvoll, zu prüfen, ob nicht auch bei Milchschokoladen die Bestimmung des Kalkgehaltes eine einfache und rasche Orientierung über die Menge der fettfreien Milchtrockenmasse ermöglicht. Falls ferner in normaler Milcbschokolade die Werte für die fettfreie Milchtrockenmasse, berechnet einerseits aus dem Kalkgehalte, andererseits aus deif ermittelten Casein annähernd übereinstimmen, würden in besonderen Fällen, etwa in Beanstandungsfällen, die nebeneinander, nach verschiedenen Verfahren ermittelten Ergebnisse sich gegenseitig kontrollieren und stützen und dadurch der Beurteilung eine erheblich sicherere Grundlage geben als bisher.

Da die fettfreie Milchtrockensubstanz aus dem Milchprotein hergeleitet wird, das sich seinerseits nach $\mathrm{Bai}$ er und $\mathrm{Neumann}$ aus dem gefundenen Casein durch Multiplikation mit 1,111 ergibt, kann man zweckmäßig so verfahren, daß man aus dem Kalkgehalte der Schokolade das Milchprotein ermittelt und die weiteren Berechnungen daraus genau nach dem von Baier und $\mathrm{Neumann}$ angegebenen Verfahren vornimmt.

Nun enthält nicht nur die Milchasche sondern auch die Kakaoasche gewisse, wenn auch erheblich geringere Mengen an Calciumoxyd. Es kann also der gefundene Kalkgehalt der Schokolade nicht direkt sondern nur unter Berücksichtigung des Kalkgehaltes der vorhandenen Kakaomasse zur Berechnung des Milchproteins verwertet werden. Ferner enthalten Milchschokoladen häufig sehr wechselnde Mengen an zugesetztem Fett und Zucker, sodaß der Gehalt an Kakaomasse erheblich schwanken kann. Um diesen Schwierigkeiten aus dem Wege zu gehen ist es daher am zweckmäßigsten, den Kalkgehalt zu Bestandteilen der Schokolade in Beziehung zu bringen, die ausschließlich der Kakaomasse bezw. der fettfreien Milchtrockensubstanz angehören und deren Mengenverhältnis in Beziehung zum Kalkgehalte als verhältnismäßig konstant angesehen werden kann. Als solche analytisch leicht feststellbaren Bestandteile kommen besonders die Gesam tasche und das Gesamtprotein der Schokolade in Betracht.

Um die betreffenden Beziehungen abzuleiten, nehmen wir einmal an, es sei a die gesamte durch Analyse gefundene Asche in Prozenten der Schokolade, $\mathbf{x}$ der auf Milchbestandtsile entfallende Anteil, die „Milchasche“, y der auf Kakao entfallende Anteil, die "Kakaoasche"; dann ist, da die gesamte Asche die Summe beider ist:

$$
x+y=a \text {. }
$$

Nun sei b der gefundene Gehalt der Schokolade an $\mathrm{CaO}$ in Prozenten, $m$ der mittlere Gehalt ron Milchasche an $\mathrm{CaO}$, n der mittlere Gehalt von Kakaomasse an $\mathrm{CaO}$, beide bezogen auf 1 , dann ist

$$
\mathrm{mx}+\mathrm{ny}=\mathrm{b} \text {. }
$$

Durch Ausrechnung beider Gleichungen und Eliminierung von y ergibt sich die Milchasche

oder

$$
\begin{gathered}
x=\frac{b-a n}{m-n} \\
x=\frac{b}{m-n}-\frac{a n}{m-n} .
\end{gathered}
$$

Diese Zeitschrift 1918, 35, 457. 
Wenn ich nun auf Grund der an verschiedenen Stellen von J. König's, Chemie der menschlichen Nahrungs- und Genufmittel ${ }^{1}$ ) angegebenen Zusammensetzung von Milch und Kakaomasse und deren Gehalt an Calciumoxyd die Mittelwerte berechne, so ergibt sich folgendes Bild:

\begin{tabular}{|c|c|c|c|}
\hline Gegenstand & $\mid \begin{array}{c}\text { Stickstoff } \\
\%\end{array}$ & $\begin{array}{c}\text { Asche } \\
\%\end{array}$ & $\begin{array}{c}\mathrm{CaO} \\
\%\end{array}$ \\
\hline Kakaomasse ${ }^{2}$ ) & 2,36 & 3,38 & 0,149 \\
\hline Milch $\left.{ }^{3}\right)$ & 0,532 & 0,72 & 0,161 \\
\hline
\end{tabular}

Hiernach beträgt also der Gehalt der Kakaoasche an $\mathrm{CaO} 4,42 \%$, der der Milchasche $22,42 \%$, also ist

$$
\begin{gathered}
\mathrm{m}=0,2242 \quad \mathrm{n}=0,0442 \\
\mathrm{~m}-\mathrm{n}=0,1800 .
\end{gathered}
$$

Setzt man diese Werte in die Gleichung 4 ein, so ergibt sich

$$
\begin{aligned}
& x=\frac{b}{0,18}-\frac{0,0442}{0,18} a \\
& x=5,56 b-0,246 a .
\end{aligned}
$$

Nun entfallen aber bei der Milch auf 0,72 Teile Asche 0,532 Teile Stickstoff; ferner wird der Proteingehalt der Milch zu $\mathrm{N} \times 6,37$ angenommen; unter entsprechender Berücksichtigung dieser Zahlen findet man dann:

$$
\text { Milchprotein }=26,1 \mathrm{~b}-1,16 \mathrm{a} \text {. }
$$

Mit anderen Worten: Die Menge des Milchproteins in der Milchschokolade findet man, indem man den Prozentgehalt der Schokolade an $\mathrm{CaO}$ mit 26,1 multipliziert und von dem erhaltenen Produkt eine Zahl abzieht, die durch Multiplikation des Prozentgehaltes der Schokolade an Asche mit 1,16 entsteht.

In ganz analoger Weise läßt sich aus den obigen Mittelwerten der Gehalt an Milchprotein aus dem Kalkgehalte und dem Gesamtstickstoffgehalte (N) der Schokolade ableiten, wobei sich folgende Gleichung ergibt:

$$
\text { Milchprotein }=21,4 \mathrm{~b}-1,35 \mathrm{~N} \text {. }
$$

Die analytische Bestimmung der Gesamtasche kann ebenso wie die Ermittelung des Gesamtstickstoffes und des Kalkgehaltes sowohl in der Schokolade direkt als auch in dem entfetteten Rückstande vorgenommen werden, wobei im letzteren Falle auf die ursprüngliche Schokolade umzurechnen ist.

Die Kalkbestimmung in Verbindung mit der Bestimmung der Gesamtasche gelingt am einfachsten nach folgendem Verfahren ${ }^{4}$ ): $20 \mathrm{~g}$ Schokolade werden in einer Platinschale in üblicher Weise vollständig verascht. Die Veraschung gelingt ohne besondere Schwierigkeiten. Die Asche wird zunächst gewogen und ergibt durch Multiplikation mit 5 den Aschengehalt der Schokolade in Prozenten.

1) Unter Berücksichtigung des in Bearbeitung befindlichen Nachtrag B zu Bd. I.

2) Mittel von 95 Proben.

3) Mittel von 705 Proben.

4) Vergl. auch Diese Zeitschrift 1918, 35, 460. 
Nach dem Wägen wird die Asche mit genau $20 \mathrm{ccm}$ Phosphorsäure (250 g sirupöse Säure im Liter) unter Umrühren mit einem Glasstäbchen übergossen, wobei die vorhandenen Kalkverbindungen in Lösung gehen. Alsdann fügt man unter weiterem Umrühren genau $20 \mathrm{ccm}$ Ammoniumoxalatlösung $(20 \mathrm{~g}$ kryst. Ammoniumoxalat im Liter) hinzu und anschließend unter weiterem Umrühren genau $20 \mathrm{ccm}$ Natronlauge (100 g festes Ätznatron in Liter). In einem Bechergläschen gibt man dieselben Reagensmengen in gleicher Weise und Reihenfolge für sich zusammen, um den Wirkungswert der Ammoniumoxalatlösung gegenüber der Permanganatlösung festzustellen.

Sodann läßt man beide Gemische unter Bedecken mit einem Uhrglase bis zum Erkalten stehen und filtriert durch ein glattes, feinporiges Kieselgurfilter. $50 \mathrm{ccm}$ der beiden Filtrate werden je nach Zusatz von $20 \mathrm{ccm}$ Schwefelsäure $(1+3)$ mit 1/10 N.-Kaliumpermanganatlösung titriert. Die Differenz der Ergebnisse beider Versuche gibt ein $\mathrm{Maß}$ für den vorhandenen Gehalt an Calciumoxyd. Bezeichnen wir die Differenz mit $\mathrm{d}$, so ist

$$
\mathrm{CaO}=\frac{100}{20} \cdot \frac{60}{50} \cdot \frac{0,005607}{2} \mathrm{~d}=0,0168 \mathrm{~d} \% .
$$

Aus dem Vorstehenden ersehen wir also, daß wir drei Wege einschlagen können, um das Milchprotein in der Milchschokolade zu ermitteln, nämlich

1. aus dem Kalkgehalte und der Gesamtasche, dem einfachsten Verfahren;

2. aus dem Kalkgehalte und dem Stickstoffgehalte der Schokolade;

3. aus dem Caseingehalte der Schokolade nach Baier und Neumann.

Es war nun von besonderem Interesse zu vergleichen, inwieweit diese Ergebnisse bei Schokoladen des Handels übereinstimmen. Einige Versuche ergaben folgende Werte:

\begin{tabular}{|c|c|c|c|c|c|c|c|}
\hline \multirow{2}{*}{$\mathrm{Nr}$. } & \multirow[b]{2}{*}{$\begin{array}{c}\text { Stickstoff } \\
\% \\
\end{array}$} & \multirow{2}{*}{$\begin{array}{c}\text { Casein nach } \\
\text { Baier und } \\
\text { Neumann } \\
\%\end{array}$} & \multirow[b]{2}{*}{$\begin{array}{c}\text { Asche } \\
\%\end{array}$} & \multirow{2}{*}{$\begin{array}{c}\begin{array}{c}\text { Calcium- } \\
\text { oxyd }\end{array} \\
\%\end{array}$} & \multicolumn{3}{|c|}{$\begin{array}{l}\text { Milchprotein, } \\
\text { berechnet aus }\end{array}$} \\
\hline & & & & & $\begin{array}{c}\text { Casein } \\
\%\end{array}$ & $\begin{array}{c}\mathrm{N} \text { und } \\
\mathrm{CaO} \\
\%\end{array}$ & $\begin{array}{c}\text { Asche und } \\
\mathrm{CaO} \\
\% \\
\%\end{array}$ \\
\hline 1 & 0,996 & 1,65 & 1,41 & 0,131 & 1,8 & 1,6 & 1,8 \\
\hline 2 & - & 0,29 & 0,89 & 0,039 & 0,3 & - & 0,2 \\
\hline 3 & - & 4,14 & 1,92 & 0,266 & 4,6 & - & 4,7 \\
\hline 4 & - & 2,72 & 1,62 & 0,186 & 3,0 & - & 3,0 \\
\hline 5 & - & 6,47 & 2,43 & 0.411 & 7,2 & - & 7,9 \\
\hline 6 & - & 2,71 & 1,68 & 0,198 & 3,0 & $\ldots$ & 3,2 \\
\hline 7 & - & 3,61 & 1,94 & 0,258 & 4,0 & - & 4.5 \\
\hline 8 & - & 3,52 & 1,99 & 0,237 & 3,9 & - & 3,9 \\
\hline 9 & 1,696 & 5,89 & 2,69 & 0,473 & 6,5 & 7,8 & 8,2 \\
\hline 10 & 1,043 & 2,71 & 1,63 & 0,192 & 3,0 & 2,7 & 3,1 \\
\hline 11 & 1,070 & 1,99 & 1,67 & 0,160 & 2,2 & 2,0 & 2,2 \\
\hline 12 & 1,505 & 3,48 & 1,70 & 0,232 & 3,9 & 2,9 & 4.2 \\
\hline 13 & 1,245 & 3.34 & 1,82 & 0,269 & 3.7 & 4,1 & 4,9 \\
\hline 14 & 1,232 & 4,41 & 1,95 & 0,320 & 4,9 & 5,2 & 6,1 \\
\hline 15 & 1,347 & 3,26 & 2,18 & 0,251 & 3,6 & 3,6 & 4,0 \\
\hline 16 & 1,271 & 6,04 & 2,39 & 0,389 & 7,4 & 6,5 & 7,4 \\
\hline 17 & 1,086 & 4,03 & 1,92 & 0,301 & 4,5 & 5,0 & 6,7 \\
\hline 18 & 0,989 & 3,04 & 1,60 & 0,249 & 3,4 & 4,0 & 4,6 \\
\hline
\end{tabular}


Bei den meisten Proben ist die Übereinstimmung hinreichend, um die nach den verschiedenen Verfahren erhaltenen Ergebnisse für die praktische Beurteilung verwerten zu können. Bei einigen Proben wurde auffallenderweise das Milchprotein, berechnet aus dem Caseingehalte, erbeblich niedriger gefunden als nach dem Kalkgehalte (Nr. 9, 13, 17). Dies kann sowohl dadurch bedingt sein, daß in die Schokolade auf irgendeine Weise Kalkverbindungen sonstiger Herkunft gelangt sind, als auch dadurch, daß sich das Casein bei der Behandlung mit Natriumoxalat nicht völlig gelöst hat; auch kann bei diesen Proben zufällig eine sehr kalkreiche Kakaomasse Verwendung gefunden haben. - Vielleicht ist es möglich, auf Grund der weiteren Untersuchung dieser Schokoladen einen weiteren Einblick zu gewinnen. Jedenfalls hat sich gezeigt, daß die Bestimmung des Kalkgehaltes bei Milchschokoladen und die Berechnung des Milchproteins daraus für die Beurteilung der Unverfälschtheit von Milchschokoladen von erheblichem Werte ist. Weitere Untersuchungen im besonderen über die gesante Zusammensetzung von Milchschokoladen des Handels sollen demnächst von uns veröffentlicht werden.

\title{
Zusammenfassung.
}

Es wird gezeigt, dif sich der Gehalt der Milchschokolade an Milchprotein bezw. fettfreier Milchtrockensubstanz aus dem gefundenen Gehalte an Asche und Calciumoxyd einerseits und Stickstoff und Calciumoxyd andererseits berechnen läßt, und daß dieses Ergebnis im allgemeinen mit dem aus dem gefundenen Casein berechneten praktisch übereinstimmt. Zur Bestimmung des Calciumoxydgehaltes in der Schokolade wird ein einfaches Verfahren angegeben.

\section{Beiträge zur Kenntnis des russischen Kochsalzes.}

\section{Von}

\author{
Prof. Mag. E. Sarin in Riga.
}

\author{
[Eingegangen am 24. September 1922.]
}

Unter den zahlreichen mineralischen Naturprodukten Rußlands nimmt das Kochsalz eine hervorragende Stellung ein. Vor dem Kriege war die Kochsalzindustrie dort stark entwickelt, sodaß die Menge dieses Produktes nicht nur für den inländischen Bedarf reichte, sondern auch ein beträchtlicher Teil ausgeführt werden konnte. Da über die chemische Zusammensetzung dieses Kochsalzes nur wenige Untersuchungen vorlagen, wandte ich mich im Jahre 1914 an die in Betracht kommenden Handelshäuser mit der Bitte, mir Proben für eine eingehende Untersuchung zu senden. Auf diese Weise standen mir 68 Proben zur Verfügung, die aus folgenden Gegenden Rußlands stammten: 